TIPA. Travaux interdisciplinaires sur la parole et le langage

$34 \mid 2018$

La langue des signes, c'est comme ça

\title{
Tribulations des langues des signes du XIXe siècle à nos jours
}

Tribulations of sign languages from the 19th century to the present day

Aude de Saint Loup

\section{OpenEdition}

1 Journals

Édition électronique

URL : http://journals.openedition.org/tipa/1976

DOI : $10.4000 /$ tipa. 1976

ISSN : 2264-7082

Éditeur

Laboratoire Parole et Langage

Référence électronique

Aude de Saint Loup, "Tribulations des langues des signes du XIXe siècle à nos jours », TIPA. TravauX interdisciplinaires sur la parole et le langage [En ligne], 34 | 2018, mis en ligne le 22 février 2019, consulté le 10 octobre 2020. URL : http://journals.openedition.org/tipa/1976 ; DOl : https://doi.org/10.4000/ tipa.1976

Ce document a été généré automatiquement le 10 octobre 2020

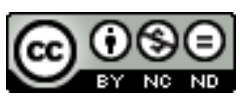

La revue TIPA. Travaux interdisciplinaires sur la parole et le langage est mise à disposition selon les termes de la licence Creative Commons Attribution - Pas d'Utilisation Commerciale - Pas de Modification 4.0 International. 


\title{
Tribulations des langues des signes du XIXe siècle à nos jours
}

Tribulations of sign languages from the 19th century to the present day

\author{
Aude de Saint Loup
}

Ce média ne peut être affiché ici. Veuillez vous reporter à l'édition en ligne http:// journals.openedition.org/tipa/1976

\section{Introduction}

2 À juste titre, les études sur l'histoire des langues des signes (LS) ${ }^{1}$ produites par les sourds se concentrent sur le XIXe siècle, lorsque l'instruction élémentaire s'est lentement généralisée dans le monde occidental et que s'est développée de manière multiforme celle des sourds. Éducation spécialisée, rassemblant artificiellement les jeunes sourds, et LS sont indissociables. Beaucoup en ont déjà éclairé les moments clés et les acteurs principaux, devenus repères classiques sinon mythiques ${ }^{2}$. On connaît peut-être moins bien les périodes transitionnelles, celles des gestations avec tous leurs possibles, qu'explorent cependant des travaux plus récents ${ }^{3}$. Les années 1830-1860 et 1970-2000, notamment, agitent toutes sortes de réflexions qui se font écho dans des contextes pourtant différents, mais montrent les permanences du questionnement autour des LS et des tensions d'objectifs et de moyens divergents. On découvre ainsi l'engagement pondéré mais déterminé de pays comme la Belgique, premier État européen à légiférer en 1836 sur l'obligation d'instruire et d'entretenir les enfants sourds-muets ou aveugles ${ }^{4}$; c'est là encore qu'en 2000 un enseignement bilingue complet (LS + français oral et écrit) et un enseignement oral avec LPC ${ }^{5}$ sont concrètement et souplement proposés en inclusion de la maternelle à la fin du secondaire à Namur. On rencontre des figures nuancées comme celle du médecin Prosper Menière ${ }^{6}$ (1799-1862) qui ne s'acharne pas sur la "réparation» médicale, comprend et respecte un mode d'être, propre aux sourds. Une histoire d'hommes, 
somme toute, inscrite dans des situations de pouvoirs (et d'impuissances) complexes et mouvantes à la surface d'un courant sociétal, scientifique et technique à vitesses variables.

Avant l'émergence d'une instruction collective dédiée, la surdité « ne » posait « qu'» un problème de communication, au-delà de réalités pratiques. Au sein d'une population majoritairement rurale, "patoisante » et illettrée, le sujet sourd pouvait trouver sa place d'actif avec plus ou moins de bonheur selon l'entourage familial et le voisinage. Des documents antiques, médiévaux et modernes témoignent d'une communication de proximité où des signes basiques se partagent entre sourds et entendants. Citant ce bon vieil exemple à l'avenir durable du Dr Alexandre Blanchet, médecin adjoint à l'Institut des sourds-muets de Paris, Prosper Menière (1853 : 114-115), médecin en chef du même Institut, le conteste : ce n'est pas parce qu'un sourd n'articule pas le mot «pain » qu'il ne se fera pas comprendre et mourra de faim. Pas plus dans les siècles antérieurs qu'à son époque et qu'aujourd'hui. Et de-ci de-là dans toute l'Europe émergent des curiosités : on éduque certains sourds à parler, lire et écrire, à l'aide de divers signes, d'alphabets manuels variés soutenant l'apprentissage de la lecture comme de l'articulation et de la lecture labiale ${ }^{7}$. Les éléments qui fondent la communication signée comme la communication orale adaptée et écrite sont quasi tous présents, latents, testés, oubliés, réinventés. En revanche, ce qu'il est convenu d'appeler les LS, au-delà des signes de proximité, imposent à leur développement une contrainte : la réunion, prolongée dans le temps, de plusieurs sourds. Ce sont donc des occasions hasardeuses de regroupement en milieu urbain ou conventuel avant le xviiie siècle, qui n'ont laissé de traces qu'en filigrane. Puis les premières "écoles ", à effectifs souvent réduits, se créent spontanément à leur intention, les rassemblent et, quand elles résistent aux fermetures, quelques-unes devenant des institutions renommées, forment ainsi des générations de sourds développant leur LS, quelle que soit la méthode appliquée.

\section{L'intégration en marche, cahin-caha}

4 Le 28 octobre 1863, Victor Duruy, ministre de l'Instruction publique, demandait aux recteurs d'académie comment introduire dans les écoles normales primaires des méthodes pour l'enseignement des sourds-muets :

... Les établissements destinés à l'éducation des sourds-muets ne peuvent recevoir qu'un très petit nombre d'élèves [...]. Le Conseil impérial de l'instruction publique [...] a émis le vœu que des dispositions soient prises à l'effet de vulgariser, au moyen des écoles normales primaires, les méthodes pour l'enseignement des sourds-muets dans les localités placées loin des centres où il existe des établissements spéciaux. Je désire, Monsieur le Recteur, connaitre votre avis sur les moyens pratiques de réaliser ce vœu. - Les écoles normales de votre ressort renferment-elles quelques maîtres qui soient initiés à une méthode d'enseignement d'une application facile, et qui puissent, à l'aide de cette méthode, former des instituteurs capables de donner l'instruction primaire aux sourds-muets? Quels seraient les moyens les plus sûrs et les plus rapides d'instruire ceux de nos maitres qui manquent de connaissances spéciales nécessaires pour se livrer à cet enseignement? Des expériences ont-elles été tentées dans votre Académie, soit par votre initiative, soit par les soins des administrations départementales, et avez-vous pu vous rendre directement compte des procédés en usage? Quelle est la méthode qui vous semblerait devoir être adoptée de préférence? Quelles modifications conviendrait-il d'apporter au programme des études des écoles normales primaires? L'introduction d'un nouvel 
enseignement présenterait-elle quelque inconvénient, et aurait-elle pour conséquence de désorganiser les études normales? (Duruy, $1863: 380-381$ ). doute faute d'avoir obtenu beaucoup de réponses, la circulaire ministérielle du 11 mars 1866 précise comment mettre en place la formation des maitres en vue de cet enseignement (Duruy, $1866: 288-291$ ).

d'années. L'âge d'entrée à l'Institut des sourds-muets de Paris, par exemple, était tardif (10 ans) et l'on estimait indispensable de préparer en amont le jeune qui aurait le privilège d'y être admis. Pour les autres, l'objectif est de rompre leur isolement et de les socialiser à minima. La recette se compose ici de signes naturels, de parole, supposant lecture labiale et articulation avec une prudente limite pour cette dernière, de lecture associée à des mots et des dessins. Les instituteurs pourraient faire ce qu'on appellerait aujourd'hui des stages d'observation, les directeurs suivre une formation à Paris (le nec plus ultra). Avec un bel optimisme, peut-être pour ne pas effrayer les recteurs d'académie, Victor Duruy affirme ces procédés simples, naturels, faciles à apprendre, non moins faciles à appliquer [...] dont l'emploi exige peu d'efforts et dont l'efficacité n'est pas douteuse. À l'instigation du Dr Alexandre Blanchet, Claude Delangle, son prédécesseur au ministère de l'Intérieur, en avait déjà fait autant en 1858, sans beaucoup plus de résultats que n'en obtiendra Victor Duruy.

Plus de cent ans après, l'intégration promue en France par la loi d'orientation de 1975 peine à être appliquée dans des conditions satisfaisantes. L'intégration « sauvage » qui se pratiquait déjà se poursuit sans dispositif d'accompagnement officiel, à quelques exceptions près, certains parents se débrouillant pour trouver un-e orthophoniste et mettre en place un soutien scolaire quand ils ne l'assurent pas eux-mêmes le plus souvent. L'accès aux études générales du second degré est un défi. Que ce soit en établissements ordinaires, relevant de l'Éducation nationale, ou dans les centres spécialisés, sous tutelle de la Santé et des Affaires sociales, la LS n'est plus utilisée dans l'enseignement, tout au plus un français signé, variable au gré des intervenants dans ces dernières structures.

8 En 1989, un rapport collectif, qui se veut décisif mais ne manque pas de paradoxes dans sa polyphonie (où les sourds sont muets, puisque absents), est coordonné par JeanPierre Bouillon, conseiller technique et pédagogique à la direction de l'Action sociale. Venu du terrain, il déclare, dès l'introduction, que l'affaire n'est pas si simple :

Qui veut s'occuper de surdité doit accepter de penser inconfortablement, de cheminer de thèses en antithèses, de changer de point de vue, d'accepter les contradictions inhérentes à cette réalité complexe (Bouillon, $1990: 8)^{8}$.

Olivier Périer, médecin qui a fondé en 1965 avec des parents d'enfants sourds le Centre Comprendre et Parler à Bruxelles, avoue dans la conclusion qu'on se confronte à plusieurs

options dont il faut faire la preuve de leur égale efficacité à long terme. Et c'est là tout le problème qui, au terme de cet ouvrage, reste entier. Car ces preuves, nous ne les avons pas. Chacun de nous a ses convictions, basées sur une expérience personnelle. Aucun de nous ne possède encore de certitudes, car les résultats des différentes options proposées n'ont pas fait l'objet d'études scientifiques comparatives. Seules des recherches rigoureuses permettront d'avancer vers des appréciations plus objectives qu'actuellement. Mais elles sont difficiles à réaliser,

TIPA. Travaux interdisciplinaires sur la parole et le langage, 34 | 2018 
puisqu'il s'agit de mesurer non pas seulement des performances mais aussi une qualité de vie. (Ibid. : 264). regarder, à se défier des apparences, des illusions faciles.

Quand il s'agit de sourds-muets, on n'improvise pas, il faut examiner beaucoup, longtemps; il faut revenir sans cesse sur ce que l'on a vu, réunir de nombreux témoignages en faveur d'une opinion, si séduisante qu'elle paraisse de prime abord (Menière, 1853 : XXIV).

11 Atermoiements que Bernard Mottez, sociologue actif dans le mouvement du Réveil des Sourds depuis la fin des années 1970, pouvait balayer d'une phrase en 1996 en tant que membre du jury à la soutenance de thèse de Christian Cuxac, premier vulgarisateur stimulant de la communauté sourde ${ }^{9}$ et linguiste chercheur, alors que se posait encore en France la question de la nature et du statut de la LS : peu importe de démontrer qu'elle est une langue, du moment que les Sourds la réclament, c'est suffisant en soi. Dans ses écrits rassemblés par Andrea Benvenuto en 2006, loin de méconnaître l'origine des débats nés de l'instruction des sourds, accompagnant le projet de création des premières classes bilingues de $2 \mathrm{LPE}^{10}$, Bernard Mottez subordonne le sujet à une affaire sociétale.

12 Aujourd'hui, à la suite de la loi de 2005 sur l'égalité des droits et des chances des personnes handicapées, il s'agit de concrétiser l'inclusion scolaire dans ses diverses modalités mais elle ne peut seule infléchir l'inclusion en société : toutes deux vont de pair. D'une manière générale, comment les Sourds qui se réclament d'une culture et d'une langue spécifiques peuvent-ils être «entendus» et vivre pleinement leurs différences ou leurs "singularités ", pour reprendre le terme que retient Hervé Benoît, revenant sur le terrain de la scolarisation, de manière plus conciliatrice dans sa volonté d'« enterrer la H de guerre », celle du « H »andicap? (Benoît, 2012: 71-73). De la réponse à cette interrogation devrait se déduire ce qui reste à faire dans tous les domaines. Et ce qui n'est pas le moindre des défis, comment édifier les passerelles d'accessibilité réciproque?

\section{Illettrisme : à qui la faute ?}

13 Les études menées jusqu'à aujourd'hui, dans le cadre scolaire et au-delà, sur la maîtrise de la lecture et de l'écriture par les sourds ou leurs situations socio-professionnelles demeurent toujours sujettes à caution. Il est difficile de collecter les données, d'établir des critères de comparaison avec un panel constant, d'avoir surtout la garantie que l'on parle bien de la même chose. Les sourds qui vivent parmi les entendants ne sont pas recensables puisqu'ils ne constituent pas un groupe social, leur existence et leur nombre sont souvent minimisés (Olivier Périer in Bouillon, 1990 : 263). Surtout s'ils vivent bien leur insertion sociale, ils ne se font pas remarquer. Mais il en est aussi qui se sont perdus dans les hôpitaux psychiatriques ${ }^{11}$.

La comparaison des résultats éducatifs entre les différents parcours, alors que s'opère une présélection dans l'orientation en inclusion ou en établissements spécialisés, mais aussi entre pays, alors que les programmes, les temps de cours, les compétences des enseignants ne sont pas les mêmes, ne facilitent pas l'évaluation (Baker et al., 2016 : $334^{12}$ ). Si l'estimation de $80 \%$ d'illettrés parmi les sourds profonds de naissance est douteuse $^{13}$ (entre sourds déclarés ou non, passés par des centres scolaires et de 
formations publics relevant de la Santé, des Affaires sociales comme de l'Éducation nationale, aux aménagements variables, ou privés, ou non scolarisés...), l'illettrisme les affecte de fait plus gravement que la population ordinaire et place la question scolaire en première ligne, mais elle n'est pas seule en cause. La construction linguistique dès le tout jeune âge, la stimulation de l'environnement familial ainsi que l'aide de techniques et d'intervenants divers jouent un rôle capital.

L'intérêt de recenser la population sourde remonte en France aux Circulaires ${ }^{14}$ de l'Institut royal des sourds-muets de Paris, entre 1827 et 1836, lesquelles lancent parallèlement un appel à mémoires sur les diverses méthodes pédagogiques en usage au-delà des frontières nationales. Après un temps de latence lié aux embarras de l'Institut parisien, elles sont suivies d'un concours au début des années 1850 afin de primer celle qui conviendrait le mieux. C'est l'abbé Charles Louis Carton, directeur de l'Institut de sourds-muets à Bruges, qui l'emportera. Mais on continuera à la chercher ensuite...

16 À la même époque, les créations d'écoles dédiées se sont démultipliées sur le territoire français, comme ailleurs en Europe et aux États-Unis, les besoins dépassant l'offre des quelques Instituts nationaux. Yves Delaporte (2016) livre, outre d'abondantes illustrations rares, de nombreuses informations sur ces écoles et leur devenir en France, essentiellement dans cette période. On y découvre, sans surprise, que l'engagement vient souvent de parents directement concernés ${ }^{15}$. Des manuels ou dictionnaires sont rédigés, et des écoles, si modestes soient-elles, ouvrent ainsi à l'initiative de pères pour leur fils (Rey-Lacroix à Montignac à partir de 1791, Alphonse Laurent à Blois dans les années 1820, Jean-Baptiste Antoine Recoing à Rumilly-LèsVaudes dans l'Aube à partir de 1825...), de sœurs (la Supérieure des Sœurs de la Charité, ayant elle-même une sœur sourde, à Tours en 1855...), de tantes, d'oncles (l'abbé Louis Marie Lambert à Paris), ou de proches à divers degrés. Ou bien ce sont des personnes elles-mêmes touchées par la surdité ou un trouble phonatoire : Adèle Cléret à Paris, M. Desongnis à Arras, Théophile Augereau (le frère Bernard des Frères de Saint-Gabriel, auteur de la phonodactylologie, un des ancêtres de la LPC) à Poitiers et Toulouse entre autres, François Laveau à Orléans, le marquis de Molleville à Ponsan-Soubiran (Gers), le père Pierre Bonhomme dans le Lot, le père A. Blain à Poitiers... Des sourds instruits dans les premiers instituts à Paris, Bordeaux et ailleurs essaiment à leur tour à Lille (Jean Massieu), Toulouse, Saint-Étienne, Lyon (David Comberry puis Claude Forestier, son gendre), Besançon, Le Puy, Vitré (Émilie et Aimée Lebreton), Nîmes, Oloron près de Pau (Pauline Larrouy), Rouen, Vizille en Isère (Joséphine et Marie Gallien) ou à l'étranger (Laurent Clerc aux États-Unis, le frère Jean-Marie-Joseph Jung - ou Young - à Montréal).

17 Dans la Deuxième Circulaire de l'Institut royal des sourds-muets de Paris, en 1829, le rapporteur, Édouard Morel, dresse un état des lieux :

Ces variations [de méthodes] prouvent que les principes sur lesquels doit reposer l'art d'instruire les sourds-muets n'ont pas encore acquis un caractère d'universalité. Trop souvent les instituteurs se sont jetés dans des systèmes exclusifs : les uns, en adoptant le langage des signes, en font un usage trop constant, et par là, tout en développant l'intelligence de l'élève, ne le familiarisent pas avec la langue écrite, parce que la syntaxe du langage mimique ${ }^{16}$ est sans cesse en opposition avec celle de nos langues artificielles, et que, dans cette lutte perpétuelle, la seconde est sacrifiée à la première; les autres, en s'attachant à l'articulation artificielle, négligent trop le développement de l'intelligence et ne songent pas que le langage des gestes, tout opposé qu'il est à la construction 
grammaticale de nos phrases, est cependant éminemment propre à provoquer le développement des idées, en figurant les circonstances qui doivent les faire naître. (Institut royal des sourds-muets de Paris, 1829 : 23.)

Il pose ainsi dès le préambule les bases d'un questionnement durable à l'origine d'une tension qui se vérifie encore actuellement et que l'on peut résumer ainsi : la LS éveille l'intelligence, développe les connaissances mais au détriment de la maîtrise de la langue nationale (orale comme écrite); le travail d'acquisition de la langue nationale est formel et néglige celui du sens. Ainsi naît la tradition des Singes et des Perroquets ${ }^{17}$. Il poursuit en formulant ce vœu :

Espérons que du conflit des diverses opinions jaillira enfin une méthode uniforme. La correspondance qui s'établit entre les institutions hâtera l'heureuse époque où tous les instituteurs, d'accord sur le but, le seront encore sur les moyens d'y arriver. Éclairés sur les résultats obtenus par d'autres moyens que les leurs, ils seront conduits à penser que la marche qu'ils ont adoptée, quoiqu'elle les mène au but, pourrait ne pas être la plus prompte ni la plus sûre ; ils seront intéressés à étudier ce qui se fait ailleurs ; ils deviendront moins exclusifs, et bientôt, l'on verra s'établir une espèce d'éclectisme dans l'art d'instruire les sourds-muets (Ibid. : 23-24).

Il faut comprendre que de cet éclectisme généralisé viendra la méthode uniforme.

Cinquante ans avant le congrès de Milan (1880), sans remonter au différend qui opposait déjà l'abbé de l'Épée, défenseur des signes méthodiques en France, à Samuel Heinicke, défenseur de l'oralisme en Allemagne, s'engage ce débat passionnel dans toutes ses graduations entre signes, quels qu'ils soient, et oral, entre exclusivisme et éclectisme, puis bientôt entre instituts spécialisés et écoles ordinaires, qui marquera le XIXe siècle et jusqu'à ces dernières années. Le formuler ainsi est simplificateur : à couleurs nationales variées, le contexte de l'instruction générale et spécifique évolue, avec les charges qu'elle suppose, la transformation des métiers suivant les phases de l'industrialisation puis de la tertiarisation, l'urbanisation et l'exode rural, l'affirmation nationaliste ou multiculturaliste ; le devenir des sourds participe de ces évolutions. La trame serait incomplète si l'on ne mentionnait pas l'impact conséquent d'avancées médicales amplifiant le pouvoir décisionnaire des médecins, le jeu d'acteurs impliqués dans l'enseignement des sourds(-muets) et une politique renforcée de contrôle ou de délégation. Édouard Morel pointe avec bon sens l'intérêt de s'entendre sur le but dont découleraient les moyens à mettre en œuvre pour y parvenir ${ }^{18}$.

Un consensus se forme néanmoins au XIXe siècle sur l'objectif de rendre les sourds-muets à la société des hommes mais pas forcément par les mêmes chemins. Quelques voix, sourdes et entendantes, modèrent l'issue, comme nous le verrons plus loin. La maitrise de l'écrit est un dénominateur commun, à l'exception de quelques «fanatiques de la parole » qui en détachent l'apprentissage du mot écrit ${ }^{19}$.

La stabilité des écoles spécialisées dépend de la relève à la disparition des fondateurs, de la compétence des maîtres, de l'adaptation aux évolutions du temps avec l'ajout de formation en ateliers professionnels par exemple, de subventions et de dons. Beaucoup $\mathrm{y}$ vont de leur promotion et de critiques à l'endroit d'éventuels concurrents. La guerre des méthodes et des personnes n'est pas nouvelle mais elle entretient un climat de violence croissante ${ }^{20}$, qui détourne des familles des centres spécialisés et alimente une désinformation autour de ce qui se fait. L'abbé Charles Louis Carton (1845: 113), en 1845 , tempère les divergences de méthodes. Il n'est rien de réellement nouveau, dit-il, dans l'histoire de l'instruction des sourds depuis Juan Pablo Bonnet (1573-1633), et 
s'il n'y a pas de méthode absolument commune à deux établissements, aucune différence essentielle ou fondamentale n'existe non plus entre les méthodes suivies dans les diverses institutions.

Il n'y a pas non plus de pédagogie commune aux professeurs d'une même institution, peut-on ajouter (Menière, $1853: 372-373)^{21} \ldots$

Entre ce qui s'écrit et ce qui se pratique, bien des écarts existent. Ainsi, poursuit l'abbé Carton (1845: 118), les Allemands, présentés comme des oralistes purs et durs, peuvent recourir aux signes, comme d'autres qui s'y disent opposés les utilisent beaucoup plus qu'on ne l'avoue. C'est en effet un brouillage répandu et durable. Le chanoine Désiré de Haerne (1865: 187), quant à lui, insiste en 1865 sur les effets néfastes de ces divisions qui encouragent des initiatives douteuses et le charlatanisme. Il revient à plusieurs reprises sur l'esprit de système et il n'est pas le seul.

\section{L'enseignement par signes remis en cause}

Publié dans la Troisième Circulaire (1832), le mémoire d'Ignace Thomas Scherr, directeur de l'Institution des sourds-muets et des aveugles de Zurich, dénonce les défauts de la communication signée chez les sourds, à combattre aussitôt qu'on aurait trouvé un autre moyen pour développer leur esprit et leur apprendre la langue. Commentant ce document, le rapporteur Édouard Morel estime bon de préciser que

L'imperfection du langage mimique ne tient pas à la nature même du langage mimique, mais seulement au défaut de culture [et que] si, dans l'Institution de Zurich, le langage des gestes est aussi pauvre que le décrit M. Scherr, il faut qu'il ait été contrarié dans son développement, ou que l'Institution ne compte pas encore un assez grand nombre d'années d'existence, et ne renferme pas une masse d'élèves assez considérable (Institut royal des sourds-muets de Paris, 1832 : 139).

Toutefois, Ignace Thomas Scherr reconnaît dans ses principes de conclusion l'utilité pragmatique des signes, sans perdre de vue que le sourd-muet doit apprendre la langue des hommes au milieu desquels il vit (troisième principe), avec l'écriture comme base d'enseignement (septième principe). La sœur d'Édouard, Octavie Morel, professeur pour les filles sourdes-muettes à l'Institut de Paris, accompagnant son oncle Degérando, s'est rendue sur place en 1830, et son rapport est publié dans cette Circulaire. Elle y confirme qu'au fond, la pédagogie mise en place à Zurich, incluant l'apprentissage de la parole, est proche de celle que défend leur oncle...

Voici cependant posées par Édouard Morel les conditions favorisant le développement des LS: un nombre suffisant de locuteurs en contact, une pratique suffisamment continue dans le temps (la LS ne s'écrivant pas), une liberté d'exister et de s'épanouir. Si d'aucuns peuvent s'étonner du retard pris ensuite par la LS en France pour s'adapter à la transmission de savoirs techniques en sciences et sciences humaines, les raisons en sont là : la LS n'a plus été un moyen de communication promu dans la scolarisation; elle est d'abord devenue par soubresauts l'auxiliaire d'un enseignement tendant vers l'oralisation, confié aux professeurs sourds de plus en plus marginalisés par le travail d'articulation imposé dans les programmes; comme il n'y avait pas encore d'orthophoniste ou de manœuvre de la parole, disait l'abbé de l'Épée, les professeurs avaient alors pour double tâche d'instruire et d'éduquer les jeunes sourds à la parole. La LS finit par être officiellement écartée, les professeurs sourds remerciés à partir de 1887.

TIPA. Travaux interdisciplinaires sur la parole et le langage, 34 | 2018 
enforçant cette exclusion, l'intégration individuelle en milieu ordinaire, devenue depuis peu inclusion, a été et continue d'être dominante ${ }^{22}$. Par-delà la volonté progressivement confirmée d'éviter la communication gestuelle, les réalités matérielles se sont imposées. Sourds et malentendants ne représentent qu'une faible partie de la population, ils sont dispersés sur le territoire et les professionnels formés aux communications adaptées (LS, pour ne pas parler du français signé, LPC) trop peu nombreux. La notion même d'égalité est à double tranchant en République française, comme le repèrent bien nos voisins suisses ou anglais : l'égalité des droits tend aussi à réduire les différences entre citoyens ${ }^{23}$ et ne s'accorde pas toujours à l'égalité des chances qui suppose des aménagements et des compensations.

L'un des arguments du Dr Alexandre Blanchet, que rapporte Prosper Menière en 1853, reste actuel :

Ne vaut-il pas mieux imposer aux 25000 sourds-muets de France l'apprentissage de la parole, que d'imposer aux 35 millions de Français parlants l'apprentissage de la mimique? (Menière, $1853: 212)^{24}$.

Une autre façon d'exprimer le malheur des natifs sourds se retrouve sous la plume de ceux qui reconnaissent la LS comme leur langue première et naturelle : ainsi, dans la Quatrième Circulaire, Édouard Morel écrit que le malheur [des sourds-muets] n'est pas qu'ils soient sourds-muets, mais que les autres entendent et parlent. (Institut royal des sourds-muets de Paris, 1836 : 832) Claude Richardin, professeur sourd-muet à Nancy, témoigne quelques pages après lui (Ibid. : 850-851) :

Le sourd-muet placé au sein de la société sent le malheur de son infirmité, par la comparaison qu'il fait entre lui et ceux qui l'entourent, mais [...] son infirmité ne lui imposerait aucune privation s'il vivait au milieu de sourds-muets. [...].

Supposons [...] une nouvelle ville en France, [habitée de] sourds-muets, [...] où personne ne parlerait [mais signerait...] J'y serais sûrement plus heureux qu'ailleurs. [...] [L'entendant y serait l'étranger parlant] montré du doigt.

Un rêve persistant que l'on retrouve en 1850 aux États-Unis, cet Eldorado des sourds vu du continent européen mais qui suit une évolution similaire à celle de l'Europe ${ }^{25}$. Les American Annals of the Deaf and Dumb (1856-1858) rapportent qu'un certain John James Flournoy a pour projet de créer un État sourd, ses pairs s'interrogeant sur ce qu'il faudrait faire alors des enfants entendants qui naîtraient de leurs couples (Padden \& Humphries, 1988 : 112)... Encore en 2005, la presse fit écho au projet de fondation d'une ville pour sourds dans le Dakota sud, baptisée Laurent en mémoire de Laurent Clerc' ${ }^{26}$, sans suite ${ }^{27}$. Sans aller jusqu'à ces projets séparatistes, Prosper Menière (1853 : 97) avait bien l'intuition des limites à respecter :

N'espérez pas attirer [le sourd] dans une société qui n'est pas la sienne. [Il] retournera de lui-même à ses compagnons d'infortune avec lesquels du moins il se sent plus à l'aise et où son amour-propre n'a rien à souffrir de la comparaison.

Il avait prévenu que les sourds-muets [...] sont moins faits pour nous que nous pour eux; c'est à nous, les riches, les favorisés, à venir vers eux, ils forment une classe à part (ibid. : 52). Un jeune sourd quittant sa famille pour l'Institut peut bien pleurer de cet arrachement, mais il découvre progressivement

qu'il se trouve dans un monde semblable à lui, il sent que ce sont des frères, il vit au milieu d'une famille qui est la sienne, où chacun pense et s'exprime comme lui, où il n'a rien à envier à personne, c'est son pays, son langage, ses mœurs, ses coutumes (ibid. : 362). 
Bénéficiant aujourd'hui de nouvelles techniques de communication visuelle et d'Internet, la communauté sourde reste en quête de lieux propres, qu'elle investit par le biais d'actions associatives, culturelles et universitaires. Sans ces outils et ces espaces identifiés, sa langue et sa culture ne pouvaient exister auparavant que dans des instituts spécialisés et des associations.

\section{Une langue (langue nationale ou LS), nécessaire mais pas suffisante}

La réciprocité d'adaptation est indispensable. S'il fallait convaincre ceux qui en douteraient encore, on doit comprendre que, même chez les personnes sourdes les mieux oralisées, la possibilité de communiquer en LS, quand elles l'ont acquise, reste importante. François Giraud, qui signe et parle, atteint d'un cancer au poumon dont il décédera peu après, explique dans l'émission que L'Eil et la Main lui a consacrée en septembre $2013^{28}$ combien il lui est difficile dans cette situation angoissante d'être sûr de bien comprendre ce que lui rapporte un médecin de ses bilans et combien il lui est pénible de ne pouvoir s'exprimer qu'à l'oral ou par griffonnage écrit. Il change d'hôpital pour cette raison, soulagé de trouver l'UNISS (Unité d'Informations et de Soins des Sourds), le pôle d'accueil spécialisé de la Pitié-Salpêtrière. Il en va de même pour une partie de ceux qui tirent le meilleur profit de leur appareillage en contours ou en implants, d'autant que la technique n'est pas sans défaillance. Qu'un contour casse, qu'un implant tombe en panne, la personne sourde doit pouvoir comprendre sans ses aides auditives. Des médecins de $\mathrm{MDPH}^{29}$ ignorent encore qu'un sourd qui parle bien reste sourd malgré tout et a besoin d'aménagements et de compensation. Des élèves parfaitement bilingues, de bon niveau scolaire, autonomes à l'écrit, pourraient réussir en intégration-inclusion individuelle (et partout) mais ils privilégient le lien avec leurs camarades sourds. Question de bien-être dans son plein sens. À l'UNISS, l'équipe est composée de professionnels sourds et entendants pratiquant la LSF, et d'interprètes en complément si besoin. Tel est le constat du Dr Alexis Karacostas, son coordinateur ${ }^{30}$ : même dans ce contexte privilégié de communication adaptée, si, par le hasard de congés et d'absences simultanés de professionnels sourds, l'un d'eux se retrouvait seul en équipe, il le vivait mal. L'outil linguistique est donc nécessaire mais pas suffisant.

Maintenant que la LS est officiellement reconnue dans plusieurs pays européens (Timmermans, 2005), proposée en principe comme langue d'enseignement à ceux qui le désirent dans le cadre d'un bilinguisme à figures variables, en France depuis la loi Fabius de $1991^{31}$ et les textes qui lui succèdent, son champ d'application reste complexe et insatisfaisant. Yves Bernard constate que nous sommes à notre tour dans une logique que nous ne maîtrisons pas, comme celles du passé (2014 : 27).

\section{Institutions spécialisées en ligne de mire}

Les instituts spécialisés sont en mutations récurrentes. On en conteste l'utilité dès le milieu du XIXe siècle: ils sont en nombre insuffisant par rapport aux besoins à pourvoir, leurs résultats ne sont pas satisfaisants, ils sont plus coûteux que les écoles ordinaires. L'Institut de Paris, pourtant célébré à sa création, n'échappe pas aux critiques de tous bords. Les dissensions internes prolongées y contribuent. Il perd 
rapidement certaines de ses élites: Laurent Clerc s'embarque dans l'aventure américaine en 1817; Auguste Bébian, promoteur entendant actif de la LS, se met à dos plusieurs collègues en critiquant leur enseignement et finit renvoyé-démissionnaire en 1821 après avoir agressé physiquement l'un d'eux. Deux ans après, Jean Massieu est également remercié pour une affaire de mœurs. Les directeurs eux-mêmes valsent à tour de rôle, la gestion générale est remise en question, la déstabilisation s'aggrave avec les modifications de projets éducatifs... Mais le reproche le plus important, à l'adresse de l'Institut de Paris comme d'autres établissements spécialisés, est d'échouer dans la mission d'origine : rendre les sourds-muets à la société des hommes en leur donnant les moyens de communiquer dans la langue nationale et leur inculquer une base d'instruction.

Cet objectif ne répond pas aux mêmes critères suivant les périodes. Instruire le plus grand nombre de sourds sans viser à en faire des savants a joué en faveur d'une communication gestuelle dans les premières décennies du XIXe siècle. Quand il ne s'agissait que de former de bons ouvriers et de bons chrétiens, comme du temps de l'abbé de l'Épée, c'était suffisant. L'abbé Carton (1845: 250) s'aligne : les sourds n'ont besoin que de connaître Dieu, leurs devoirs et un métier. Comme il n'y a pas si longtemps encore, des professionnels de l'accompagnement en collège ordinaire estimaient absurde d'orienter des sourds profonds en filière générale au lycée. À quelques privilégiés au XIXe siècle était alors réservé l'apprentissage du français oral (lecture labiale et articulation), qui se pratiquait déjà dans le cadre du préceptorat et ne pouvait être dispensé par la suite qu'en petits effectifs, ce qui n'était pas le cas à l'Institut de Paris dont la classe d'articulation était surchargée et le fonctionnement hebdomadaire insuffisant. L'écrit (lecture et expression écrite) pouvait s'adresser à tous, mais il était nécessairement basique pour ceux qui signaient, affiné pour ceux qui parlaient ou s'efforçaient de le faire, d'autant que certains en contestaient la parfaite maitrise à partir des signes, autre point de division toujours d'actualité ${ }^{32}$. C'est pourtant un lien fort établi par les premiers instructeurs usant de la mimique, de signes divers, et qui continue de faire l'objet de recherches ${ }^{33}$. On observe de jeunes élèves dont la LS est la langue première aborder la lecture d'un texte nouveau en l'accompagnant de signes discrets, à la façon dont un jeune entendant déchiffre mots et phrases en articulant à voix basse. Des orthophonistes repèrent que certains élèves signeurs et bons lecteurs, hermétiques à la phonétique, ne comprennent pas les transcriptions sous ce mode alors que le sens du texte correctement orthographié leur est clair ${ }^{34}$.

Parallèlement, le regroupement des uns et des autres en un même lieu inspire des critiques. On dénonce les instituts spécialisés comme une fabrique de sourds-muets, certains en sortant plus sourds et plus muets qu'ils n'y étaient entrés ${ }^{35}$. La communication signée que les élèves développent librement entre eux, soutenue par leurs professeurs sourds, échappe aux professeurs entendants. L'écart d'incompréhension grandissant, les sourds signeurs rejetteraient la société des entendants $^{36}$, tandis que ces derniers, en particulier les médecins dont le rôle émergeant au milieu du XIXe siècle s'accentuera ensuite, dénoncent leur incapacité à maitriser les langues nationales, orales comme écrites, et finissent par les assimiler aux idiots ou arriérés à la fin du XIXe siècle et au début du suivant. Dépité d'avoir dû quitter l'Institut dont il attendait une promotion, Auguste Bébian a eu tôt sa part de responsabilité dans l'affaire, attaquant le système de signes grossiers et obscurs (ibid.: 121-122) ou signes méthodiques, sorte de français signé complexifié par son parrain, 
l'abbé Roch-Ambroise Cucurron Sicard, et ses piètres résultats. Sa critique est élargie malgré lui à la LS en général. C'est encore lui qui répand l'idée, exploitée par d'autres, que Jean Massieu, vedette de Sicard qui l'avait fait venir avec lui de Bordeaux et qu'il « exposa » régulièrement, lit en réalité avec peine des textes pour enfants. En outre, la mixité des filles et des garçons encourage des mariages entre sourds qui reproduisent ainsi en partie leur infirmité.

\section{Division des sourds-Sourds, de l'éclectisme à l'exclusivisme}

De là naît la volonté de classer et séparer les élèves suivant leur degré de surdité, leur âge pour éviter le contact des plus jeunes avec les plus anciens, et leur sexe. Pour finir, quelques-uns, dont le nombre ira croissant, pour ces raisons et pour d'autres, estiment nécessaire d'intégrer les sourds en milieu ordinaire. Si les signes s'attrapent par contact, peut-être bien que la fréquentation des parlants serait également contagieuse... Tout au moins le comportement s'alignerait par imitation, comme le suggérait Rémi Valade-Gabel, avec l'idée de fondre les sourds-muets dans la grande famille humaine, telle que la rapporte Désiré de Haerne qui s'empresse d'ajouter qu'on veut les mêler généralement aux autres enfants pour les rendre plus sociables, et c'est ordinairement un résultat contraire qu'on obtient (de Haerne, 1865 : 225).

Jean-Marc Gaspard Itard, médecin en chef à l'Institut de Paris de 1800 à 1838, est à l'origine des premiers classements de sourds en France, distinguant principalement ceux de naissance et les plus atteints de ceux qui l'étaient devenus après acquisition de la parole et conservant des restes auditifs. Pour les premiers, le travail de l'articulation serait une épreuve inutile, à quelques exceptions près, alors qu'il conviendrait de le développer pour les seconds. Cette distinction a été mise à profit pour casser certains mythes : Laurent Clerc et Ferdinand Berthier étaient des devenus-sourds. Jean Massieu, sourd de naissance, prétendait-on à la suite d'Auguste Bébian, lisait des textes formulés simplement. Laurent Clerc et Jean Massieu ont été instruits par la voie des signes méthodiques complexifiés, sorte de français signé difficilement praticable, de l'abbé Sicard. Promoteur d'une LS dégagée de la méthodique, l'action d'Auguste Bébian a été de courte durée. L'enseignement en LS ne pouvait plus être assuré que par des professeurs sourds qu'il avait formés, les entendants usant plutôt de la méthode mixte, plus courante à Paris comme ailleurs. Et ce terme de mixte recouvre de fait des réalités très disparates.

41 Dans sa conclusion à la Troisième Circulaire, Édouard Morel tente de synthétiser les diverses pratiques qui varient suivant les pays aussi bien qu'à l'intérieur des frontières, et il est bien placé pour le voir mais effleure le sujet en ne signalant que l'éviction des signes méthodiques, au sein d'un même institut. Il ajoute qu'en dépit de cela

Toutes [les Institutions] obtiennent des résultats plus ou moins satisfaisants : c'est qu'il faut le reconnaître, la méthode consiste moins dans l'adoption de tel ou tel instrument de communication, que dans l'emploi que l'on en fait pour opérer la génération des idées. [...] Avant d'emprunter à une Institution son mode d'enseignement, il ne suffit pas de constater les résultats qu'elle obtient, il faut encore examiner sa situation et les conditions auxquelles elle obtient ces résultats ; conditions sans lesquelles on attendrait vainement le même succès (Institut royal des sourds-muets de Paris, 1832 : 352). 
42

en garde contre l'usage exclusif des signes comme de la parole, les deux ayant leur utilité. Si le nombre des instituts augmente, il demeure insuffisant, d'où l'intérêt de former des instituteurs dans des écoles normales comme pourrait le redevenir officiellement l'Institut de Paris, et de conseiller les parents sur la façon de commencer eux-mêmes à éduquer et instruire leur enfant.

Pour clore la présentation de l'Institut de Saint-Pétersbourg au début de la Quatrième Circulaire, le même rapporteur, en accord avec les directeurs russes, alerte de nouveau contre un système exclusif, le meilleur mode d'enseignement n'est pas toujours le plus méthodique (Ibid., 1836: 386). Sa sœur lui fait écho un peu plus loin, rapportant que la méthode allemande est plus complète, plus logique, et surtout plus uniforme que la nôtre; et que nos procédés sont infiniment supérieurs à ceux des professeurs allemands. Où l'on découvre que l'enseignement de la parole est privilégié parce que les Allemands sont peu doués pour le mime, mais aussi parce que la langue parlée y est plus «rude » et donc plus accessible que le français par exemple... Cette idée sera reprise par l'abbé Carton, en $1845^{37}$. Son compatriote, le chanoine Désiré de Haerne, directeur de l'Institut de Bruxelles, explique de son côté que l'accès à l'écrit est plus facile en allemand, tandis que le français et l'anglais ne s'écrivent pas tout à fait comme ils se prononcent (de Haerne, 1865 : 131). Néanmoins, poursuit-il,

L'association des divers signes représentatifs de la pensée, signes naturels, méthodiques, arbitraires, dactylologiques, syllabiques, écrits ou articulés est un phénomène qui ne se présente pas seulement dans l'éducation, mais qui exerce son influence dans les relations sociales en général, souvent pendant tout le cours de la vie. C'est ce qu'ont perdu de vue la plupart de ceux, Allemands et autres, qui prétendent suivre une méthode fondée sur un principe unique, tandis que la nature, surtout chez les sourds-muets, est complexe et relève, à des degrés différents chez les divers individus, des lois qui régissent les facultés intellectuelles et physiques de l'homme dans leurs nombreux rapports réciproques ${ }^{38}$.

Prosper Menière insistait également sur l'intérêt à ne se priver d'aucun moyen :

Ce ne sont pas les méthodes qui sont exclusives, mais bien les hommes qui les appliquent. [...] Pour nous, notre conviction est bien arrêtée, et, malgré l'autorité des hommes éminents qui se sont prononcés contre le mélange des deux systèmes, nous persistons à penser qu'ils peuvent et qu'ils doivent même être employés simultanément dans beaucoup de cas, et que ce serait le moyen de rendre l'éducation des sourds-muets beaucoup plus complète; car, possédant le langage oral et celui des signes, ils pourront employer l'un ou l'autre mode de communication, selon les circonstances où ils se trouveront. [...] Conserver ces deux modes d'instruction; nous voudrions qu'il en existât un troisième, un quatrième. (Menière, 1853 : 118, 171 et 391).

Les protestants européens suivent cependant de préférence la méthode allemande, jusqu'à Saint-Hippolyte-du-Fort (Gard), ce qui complique la tâche, la langue étant française. Un professeur sourd de l'Institut de Bordeaux reproche à ces mêmes Allemands de croire que la parole est indispensable pour l'acquisition des idées. C'est une erreur et des plus capitales; j'aurais envie de leur envoyer une colonie de perroquets pour leur pénitence (Institut royal des sourds-muets de Paris, 1836 : 510). L'Angleterre, les ÉtatsUnis, la France et les catholiques en général adoptent les signes, suivant des procédures variées dans cette première moitié du XIXe siècle. L'Italie est aussi chaudement recommandée : l'expressivité gestuelle y est spontanée, tout le monde parlant avec les mains. Dans la Troisième Circulaire Giovanni Battista Scagliotti, instituteur de sourdsmuets à Turin, mentionne cependant l'apprentissage de la parole mais revient sur les

TIPA. Travaux interdisciplinaires sur la parole et le langage, 34 | 2018 
qualités des signes et se prend lui aussi à rêver qu'il se forme une académie de ce langage universel qui, étendu à tous les pays, permettrait même aux entendants de voyager sans avoir besoin de connaître les autres langues (Institut royal des sourds-muets de Paris, 1832 : 396). Quelques décennies après, ces appréciations n'ont plus cours, Milan chante la parole pure ${ }^{39}$.

Le désir d'instaurer une éducation orale fait son chemin partout, et il se monte des expériences pour développer l'articulation, jusqu'à l'Institut de Paris comme aux ÉtatsUnis. Sans entrer dans le détail des variations dans le temps et dans l'espace, des réformes et des coups d'arrêt, l'éclectisme "raisonnable ", défendu par une majorité d'acteurs tout au long du XIXe siècle, est voué à une condamnation radicale dans quelques pays d'Europe au profit d'une oralisation exclusive à terme ${ }^{40}$. On notera toutefois que, même là où l'oral s'impose, il demeure souvent un interprétariat en LS lors de discours et de grandes réunions ${ }^{41}$. L'argument qui l'aurait emporté, à côté de considérations plus matérielles et politiques, est que la LS est un obstacle à l'apprentissage de la parole et que cette dernière est prioritaire pour l'accès à l'écrit, à l'instruction, à la société. Cette incompatibilité rend la mixité impossible.

\section{LS, le retour}

Quand, dans les années 1970, suivant l'impulsion américaine et la montée des revendications en faveur des minorités, l'éducation oraliste est rendue responsable de l'illettrisme des sourds, de leurs échecs scolaires et en insertion socio-professionnelle ${ }^{42}$, le retour des LS se fait progressivement, au travers d'initiatives marginales. La communication totale (toutes les formes de communication mêlées) se développe d'abord aux États-Unis pour tendre ensuite vers le bilinguisme. Les premières classes bilingues en France sont créées par Danièle Bouvet, orthophoniste, en 1979 à l'école maternelle de Bossuet à Paris, qui s'associe Marie-Thérèse L'Huillier, sourde signante ${ }^{43}$, toutes deux présentant en parallèle l'émission Mes Mains ont la parole pour la deuxième chaîne de télévision, des contes signés pour enfants, doublés d'une voix off. Annette Gorouben, orthophoniste, fonde en 1981 à Paris le CAMSP de l'Institut Arthur qui devient deux ans plus tard le CEBES (Centre Expérimental Bilingue pour Enfants Sourds), œuvrant avec des professionnels entendants et sourds pour l'éducation multimodale des tout jeunes enfants et l'accompagnement de leurs parents. En 1990 y sont admis des enfants entendants de parents sourds. Les professionnels ont gardé cette volonté de créer des espaces où circule la parole, quelle que soit la langue, pour faire du lien entre les deux mondes et les deux cultures (Philippon-Pointereau, 2013 : 65). L'association 2LPE ouvre ses propres classes bilingues à l'école Paul Fort de Poitiers en 1984, tandis que se créent des cours de LS, la LS étant également diffusée sous forme théâtrale par $\mathrm{IVT}^{44}$. À l'émission La Marche du Siècle, consacrée au «Peuple des sourds » en $1992^{45}$, deux intervenants sourds sont sur le plateau : l'un qui signe, Daniel Abbou; l'autre qui parle, Joël Chalude. Ce dernier explique que, bien que connaissant et pratiquant la LS, sa langue naturelle, il préfère parler, le français étant sa langue maternelle, pour pouvoir s'exprimer plus précisément qu'il ne pourrait le faire en LS. Il corrige ce qui pourrait être compris de manière négative : la LS est riche mais elle doit rattraper un grand retard depuis son ostracisation en France ${ }^{46}$. Deux centres pionniers d'accompagnement social, le CPSAS (Centre de Promotion Sociale des Adultes Sourds) à l'INJS (Institut National des Jeunes Sourds) de Paris, en 1980, et le SERAC (Sourds 
Entendants Recherche Action Communication), en 1986, proposent parallèlement les premiers interprètes officiels, suivis en 1993 par l'ESIT (École Supérieure d'Interprètes et de Traducteurs) qui ouvre une section LS de formation exigeante afin de développer l'interprétariat dans l'enseignement et les branches les plus spécialisées.

La Suède est le premier pays européen à reconnaître officiellement la LS en 1981 et à développer le bilinguisme sans oral (LS + suédois écrit, un « dilinguisme »?), les sourds recourant après l'école au service d'interprètes. La mixité linguistique est cependant adoptée par la plupart des pays qui veulent s'émanciper de l'éducation strictement orale des sourds dont le maintien reste toutefois conforté par les progrès prothétiques et l'essor de l'implant cochléaire.

Ce tiraillement est perceptible dans le Rapport Bouillon de 1989, affirmant conjointement la priorité de l'accessibilité communicationnelle (LS, LPC) et la primauté de la langue française écrite et parlée, dont l'acquisition est un droit ne pouvant être refusé aux sourds ${ }^{47}$. La notion de bilinguisme est encore à définir et le rapporteur s'oppose à la recette du pâté d'alouette: un cheval de LS pour une alouette de français (Bouillon, 1990 : 32). Les parents de Magali témoignent ensuite, à la recherche d'une petite alouette de LS. Aucune possibilité de scolarisation bilingue n'existe près de chez eux et ils se sont démenés pour mettre leur enfant en contact avec des adultes sourds. C'est ce qui lui a permis de s'ouvrir puis de rentrer parallèlement dans l'apprentissage du français. Tout à fait bilingue, stimulée de tous bords, Magali qui a finalement été scolarisée au CEOP (Centre Expérimental d'Orthophonie et de Pédagogie) puis en filière générale scientifique au Cours Morvan, a obtenu en bout de course son diplôme de pharmacie. Une demande d'interprète avait été faite la première année universitaire mais elle y a renoncé au bout de quelques mois car, en 2000, le décalage entre la LS et le niveau technique requis dans ce domaine était encore trop grand. Magali a donc appliqué le système $\mathrm{D}$ des étudiants sourds qui l'avaient précédée : se lier à d'autres étudiants, obtenir des notes de cours et un soutien de la part de certains professeurs, lire, lire, lire...

50 Dans «Le Peuple des sourds » (1992), Yvette Thoua-Zegers de Beyl, mère belge d'une jeune sourde très profonde, explique un peu de la même manière son cheminement en bilinguisme "sauvage", avec toutefois l'apprentissage du français soutenu par la LPC. Sa fille Marie, comme Magali, a poursuivi une scolarité en études supérieures. Depuis, toujours très impliquée dans l'accessibilité scolaire, Yvette Thoua-Zegers de Beyl a cofondé dans la communauté scolaire Sainte-Marie de Namur une structure bilingue complète, le français oral n'étant pas exclu pour les sourds, la LS étant ouverte aux entendants ${ }^{48}$. L'établissement mêle des jeunes entendants, des jeunes sourds oralisants appareillés, voire implantés, (avec LPC possible) et signeurs. Rien n'est obligatoire, tout est possible, suivant l'appétence de chacun : un luxe! Ce n'est pas exactement ainsi qu'est conçu le bilinguisme dans les quelques centres les plus actifs en France, soutenus par l'ANPES, association de parents engagés, où l'oral est optionnel, l'appareillage exceptionnel. Mais des expériences se sont faites et se font de manière plus discrète et souple $^{49}$, dans le Primaire essentiellement, pas assez encore cependant, un effort particulier restant à développer dans le second degré où interviennent des centres médico-éducatifs en compensation suivant leurs disponibilités et l'accueil des établissements. À défaut, ce sont des interprètes ou des interfaces qui se déplacent et facilitent le suivi des cours en décalant cependant le lien pédagogique. 


\section{Une minorité signant et/ou parlant difficilement, qui dérange}

51 Est-on réellement dans un processus de "colonisation », d'oppression d'une minorité ? Est-ce l'amélioration des aides auditives qu'il faut condamner ou leur application généralisée et inadaptée à ceux qui ne peuvent en tirer profit ? Le travail de lecture labiale et d'articulation est-il une torture inutile ? Des extrémistes s'illustrent comme le Dr Ling, promoteur de l'oralisme au Canada qui, dans un article de 1999, rattache la méthode gestuelle à un modèle du XIXe siècle et proclame que la sienne mettra fin à la "Communauté des Sourds" (will put an end to the "Deaf Community"; Lachance, $2007: 251$ ). À l'opposé, on dénonce le dépistage précoce de la surdité et les dérives de la recherche génétique pouvant mener aux sélections embryonnaires ${ }^{50}$. Quand dès le XIXe siècle, dans le sillage du courant eugéniste, on prévient contre les mariages entre sourds ${ }^{51}$, qu'on pratique ensuite leur stérilisation dans quelques pays européens au-delà de la Seconde Guerre mondiale, sans doute, oui, le risque est là.

Prosaïquement, la société entendante, elle, n'a pas besoin de la LS ; elle sait s'inventer des codes gestuels en situations particulières (la bourse, la plongée sous-marine, dans les airs maintenant, etc.) mais pas plus. Si la surdité peut affecter n'importe lequel de ses membres, elle touche en majorité des devenus sourds qui conservent la faculté de parler, particulièrement des presbyacousiques, quoique le phénomène concerne de plus en plus des jeunes adeptes de musique électronique et même certains DJ célèbres ${ }^{52}$. Cependant les sourds nés sans avoir acquis naturellement la parole et ce qui en découle, existent, et leurs parents, de tous milieux sociaux, géographiques, de toutes religions ou athées, ne peuvent pas être indifférents à leur sort, sauf exceptions. Si le "métier " parental est d'accepter la personnalité propre de l'enfant et d'accompagner son cheminement personnel, le besoin de communication en proximité est compréhensible, la crainte des difficultés qu'il rencontrera à faire de véritables choix de vie en société est légitime. La solution est donc bien celle de l'information sur les moyens d'adaptation en réciprocité. Jusqu'où les sourds peuvent-ils s'adapter sans se "désintégrer ", jusqu'où la société peut-elle développer ses accessibilités que la seule contrainte législative rend factices?

Il ne suffit pas, en effet, de chiffrer le nombre des scolarisés ou des embauchés : quels espaces de vie et de fonctionnement peut-on ouvrir en intelligence? Trop de jeunes et d'adultes sourds, oralisés ou signeurs, demeurent frustrés, isolés ${ }^{53}$, oubliés, notamment dans les promotions en entreprise. Il manque encore bien des réflexes, malgré des sensibilisations initiales et une proximité quotidienne. Fêtes familiales et réunions professionnelles, quand on ne dispose pas d'un système de visiocommunication, d'interprètes ou d'interfaces, demeurent des épreuves pour les sourds. C'est un travail de longue haleine, dont certaines solutions partielles peuvent être utiles à d'autres (préparation d'écrits, projections, transcriptions simultanées), mais c'est avant tout une affaire d'attention constante et d'ouverture pour casser les automatismes en communication directe. L'un des avantages de la LS est d'ailleurs qu'elle se voit, elle alerte; et si le sujet sourd n'est pas inhibé, il sait se manifester autant qu'il faut pour se rappeler aux autres. 


\section{Bilinguisme offensif, défensif, ou vers un biculturalisme du partage}

54 L'inclusion scolaire, bon an mal an, finit par s'imposer en Europe dans les années 2000. Les établissements spécialisés ferment en Grande-Bretagne, en Italie, en Grèce, où les situations de scolarisation en milieu ordinaire ne se ressemblent pas. La formation des enseignants favorisant l'inclusion diffère d'un État à l'autre; l'accompagnement de compensation peut être aléatoire du fait de la dispersion des élèves sourds encore souvent inclus individuellement, et par manque de professionnels comme de financements. L'oral prime partout, même dans des établissements spécialisés maintenus par certains pays comme en Flandres, en Russie (qui a reconnu la LS en 2012). Le développement de l'implantation cochléaire renforce cette orientation qui inquiète toujours la communauté sourde et ses sympathisants. Les modalités éducatives à l'endroit d'élèves sourds restent multiples et changeantes, tant et si bien que la Suède elle-même a réintroduit récemment l'enseignement oral pour les sourds. Les formes de bilinguisme sont également très diversifiées : deux intervenants dans la même classe dispensent simultanément le cours à l'oral ou en LS ; ou bien une partie des cours se fait à l'oral, une autre en LS. Un constat partagé pointe le manque de formation de haut niveau en LS, chez les sourds comme chez les entendants. Si l'on ne conteste plus guère que la LS aide les sourds profonds à acquérir une langue, à s'impliquer davantage en classe, à échanger les informations, à entrer dans la lecture décryptée en signes, cependant, des quelques études menées jusqu'à aujourd'hui, l'impact de ce programme de lecture ne ressort pas clairement (Baker et al., 2016 : 332-334).

Quoique des insatisfactions demeurent et qu'il reste donc à faire, les conditions ont profondément évolué. En France, il fallait bien un temps de reconstruction à la LS pour sortir des circuits confidentiels, familiaux ou associatifs, et gagner la reconnaissance qui lui permet de se manifester dans tous les domaines. L'interprétariat est de plus en plus qualifié et spécialisé. Lorsqu'ils restent en lien avec leurs pairs, certains sourds oralisés qui ont réussi en études supérieures et se sont insérés à bon niveau dans la société à partir des années 1960 (et même avant, si l'on songe à de fortes personnalités telles que Suzanne Lavaud, docteur ès lettres en 1924) sont parmi les plus actifs à œuvrer au développement de la LS et à l'extension de son usage. Plus récemment, des sourds signants dépassent le cadre médiatique et culturel en obtenant des postes universitaires ou de chercheurs au CNRS. Le droit d'enseigner leur est restitué (mais pas toujours celui d'exercer dans des conditions acceptables...). Des entendants agissent à leurs côtés. Les textes législatifs confirment des avancées, au moins de principe ${ }^{54}$. C'est ensuite, et comme cela l'a toujours été, l'implication des uns et des autres qui en permet les réalisations. On se plaint beaucoup en France de la pesanteur administrative; mais il se trouve aussi des personnes attentives aux situations particulières, s'efforçant de chercher des solutions, au sein même de l'Éducation nationale. Des responsables académiques s'engagent, ainsi que des référents et des médiateurs. Sans se leurrer ni tomber dans un angélisme attentiste, il y a de quoi construire ${ }^{55}$.

La communauté sourde n'existe pas naturellement. Elle est née d'institutions regroupant les sourds, les situations géographiques d'isolement collectif étant exceptionnelles, comme dans l'île de Martha's Vineyard avant le développement du tourisme. Elle n'a pas de territoire originel, c'est une diaspora d'histoire récente, sans 
tradition scripturaire ni transmission familiale à plus de $90 \%$ (la proportion de parents entendants d'enfants sourds). En dépit de ces conditions fragilisantes, elle résiste et s'impose avec plus de succès dans un biculturalisme ouvert.

Le nombre des sourds bilingues-biculturels qui signent, lisent, écrivent et parlent éventuellement, est croissant; ce sont les meilleurs passeurs. Certains excellent dans l'art de la conciliation, sachant emmener l'entendant au but recherché. Des élèves peuvent se révéler maîtres en pédagogie auprès de leurs professeurs et de leurs camarades. Ces bilingues nous apprennent notamment, à nous entendants, les limites entre ce qui se partage et ce qui nous échappe. Le mode d'être sourd, ou la culture sourde suivant la définition anglo-saxonne, était déjà bien perçu par des entendants au XIXe siècle, alors que la communauté sourde était plus vulnérable et que ses membres ne disposaient pas des moyens de communication actuels, une fois sortis des institutions, pour l'entretenir régulièrement. Comprendre ces singularités et les respecter conduit à nuancer les interventions en évitant un acharnement mal ciblé, peu efficient à terme sinon destructeur, quand il n'alimente pas des réactions de rejet. Des peurs persistent malgré tout de part et d'autre : celle d'un deuil à faire du semblable, celle d'être menacé dans son identité, celle de ne pas savoir faire réciproquement. Elles se répercutent sur le plan linguistique. La LS nuirait à l'apprentissage des langues nationales, et vice-versa. Et que dire des pidgins, des français signés et de leur dernierné, le FCFC (Français Complet Signé Codé), dont on ne sait pas très bien ce qu'il devient d'ailleurs? Avoir pour seul objectif d'apprendre aux sourds les langues nationales, même via le bilinguisme, viserait à rendre le Sourd le plus entendant possible (Lachance, 2007 : 257). Pour d'autres, songeant surtout à l'écrit, c'est armer le sourd pour qu'il gagne l'autonomie et l'indépendance permettant que des décisions ne se prennent pas à sa place. Averti par l'expérience dont il offre un bouquet final sous forme de chroniques, Marc Renard, devenu sourd à deux ans, enfourchait régulièrement ce cheval de bataille dans ses interventions et ses écrits (Renard, 2016). Sa seconde activité professionnelle d'éditeur spécialisé en surdité, où des manuels de lecture labiale peuvent côtoyer des livres sur la LS, l'histoire des sourds, des BD, des romans, invite aussi tous les sourds (et leurs amis entendants) à lire...

Malgré tout, les zones de partage s'étendent, ce qui est un facteur d'apaisement encourageant la collaboration tant que l'on ne l'impose pas sans préparation et qu'elle s'accomplit sur la base d'un volontariat dans un « consentement éclairé ». Si la bonne volonté et les efforts d'un sourd pour maîtriser la langue nationale, ceux d'un entendant pour entrer dans la LS ne suffisent pas toujours, cela n'empêche pas la communication qui ne se réduit pas à une langue bien oralisée ou bien signée ${ }^{56}$. L'enfant sourd et ses parents entendants n'ont pas de temps à perdre pour l'engager, quitte à l'améliorer progressivement ${ }^{57}$. En outre, et pour revenir au champ scolaire, Désiré de Haerne faisait déjà remarquer que

[...] la liberté néanmoins doit prévaloir en matière de méthode ; l'instituteur doit être dirigé mais non contraint [...]. Un bon instituteur ayant une méthode vicieuse vaut mieux qu'un mauvais instituteur avec la meilleure méthode possible. Et d'ajouter, on dirait, à lire certains auteurs, que les élèves sont faits pour les instituteurs plutôt que les instituteurs pour les élèves (de Haerne, $1865: 230$, notes 161 et 170).

59 Par méthode vicieuse, il faut comprendre du bricolage, tout comme l'on signote, pouvait dire Bernard Mottez, ou l'on s'exprime hors académisme face à la grande hétérogénéité des acquis d'élèves de toutes catégories, et plus encore d'élèves sourds, à la fin du 
premier degré. Il est évident que tous les outils disponibles sont utiles. Mais le désir de rencontre qui conduit à relativiser le savoir, à défaire les dogmes ${ }^{58}$, est le déclencheur principal. Un professeur n'est pas un « rééducateur » linguistique, ce rôle incombant désormais à des professionnels tels que les orthophonistes, bien que celui qui enseigne plus spécifiquement une langue, connaissant ces techniques, gagne en efficacité ${ }^{59}$. En revanche, toute discipline d'enseignement est confrontée à son expression écrite en français. Le professeur intervient donc dans la maîtrise du lexique technique comme de la formulation. Mieux vaut cependant connaître les particularismes liés aux surdités pour éviter de sanctionner d'office une copie d'élève. Et en communication, sans être puriste, connaître des bases dans la langue de l'élève, même si certains pédagogues doués sont capables de transmettre sans cela, et certains élèves doués capables de recevoir sans cela...

De ce rapide tour d'horizon, il apparaît que chaque coup de balancier est précédé et suivi de questionnements et d'expériences produits en sable mouvant, dont les traces s'effacent pour être réinventées ensuite. Le sujet sourd en a été plus souvent l'objet qui subit que l'acteur qui décide. Il y a eu pourtant de belles conquêtes remportées par les Sourds au XIXe siècle. Ainsi, en France, Ferdinand Berthier obtient pour ses pairs le droit de vote en 1848, quand les femmes devront attendre 1944. Toutefois le droit d'enseigner leur a échappé ${ }^{60}$. Il revient actuellement, anticipé par la présence d'éducateurs sourds d'abord, avec des professeurs sourds diplômés, à condition de trouver le dispositif pour l'exercer. Il convient de rappeler sans polémiquer que l'implication, à tous niveaux de la société, de sourds issus de l'oralisme y est pour quelque chose aussi ; les preuves sont faites de leurs compétences et une partie d'entre eux peuvent justifier leur besoin de LS, si bien oralisés soient-ils. À braquer le projecteur de poursuite sur une modalité linguistique ou sur une finalité unique, on laisse dans l'obscurité le reste du plateau de la vie sans mesurer ce qui s'y joue, s'y gagne ou s'y perd. Qui perd gagne en éclairant la scène, en se défaisant d'habitudes et de préjugés pour d'autres possibles.

\section{BIBLIOGRAPHIE}

Baker, A., van den Bogaerde, B., Pfau, R. \& T. Schermer (2016) The Linguistics of Sign Language. An Introduction, Amsterdam \& Philadelphie: John Benjamins Publishing Company.

Baynton, D. C. (1996) Forbidden Signs. American Culture and the Campaign against Sign Language (Signes interdits. Culture américaine et la campagne contre la langue des signes), Chicago \& Londres: The University of Chicago Press.

Bell, A. G. (1884) Memoir upon the Formation of a Deaf Variety of the Human Race, Washington D.C.: National Academy of Sciences.

Benoît, H. (2012) Pluralité des acteurs et des pratiques inclusives : les paradoxes de la collaboration, La Nouvelle Revue de l'Adaptation et de la Scolarisation, 57, Suresnes: INSHEA, p. 65-78. 
Bernard, Y. (2014) L'Esprit des Sourds. Les signes de l'Antiquité au XIXe siècle, Les Essarts-le-Roi : Éditions du Fox.

Bouillon, J. P. dir. (1990) La surdité chez l'enfant en France, Paris : CTNERHI.

Brugeille, J. P. (2003) L'enseignement de la LSF dans le cadre d'un enseignement en langue des signes au collège, Langue Française, 137, 1, 2003, p. 60-69.

Buton, F. (2009) L'administration des faveurs. L'État, les sourds et les aveugles (1789-1885), Rennes : Presses universitaires de Rennes.

Cantin, Y. (2014) Les Sourds-Muets de la Belle Époque. Une communauté en mutation, Thèse de doctorat en Histoire, EHESS, Paris.

Cantin, Y. (2017) La Noétomalalie Historique [en ligne]. Disponible sur : https:// noetomalalie.hypotheses.org/author/noetomalalie (consulté le 23 juillet 2017).

Carton, C. L. (1845) Mémoire en réponse à la question suivante « Faire un exposé raisonné des Systèmes etc. ", in Tome XIX des Mémoires Couronnés et Mémoires Des Savants Étrangers, 1845-1846, Bruxelles : Académie Royale de Belgique (iBooks de Google).

Cuxac, C. (1983) Le Langage des Sourds, Paris : Payot.

Degérando, J.-M. (1827) De l'éducation des sourds-muets de naissance, Tome premier, réédité in Renard M. \& M. Renard, Bibliothèque sourde, Les Essarts-le-Roi: Éditions du Fox.

De Haerne, D. (1865) De l'enseignement spécial des sourds-muets, considéré, dans les méthodes principales, d'après la tradition et le progrès, Bruxelles, réédité in Renard, M. \& M. Renard (2015) Bibliothèque sourde, Les Essarts-le-Roi: Éditions du Fox.

Delaporte, Y. (2016) L'École des sourds, Les Essarts-le-Roi: Éditions du Fox.

Duruy, V. (1863) Demande de renseignements sur l'introduction dans les écoles normales primaires de méthodes pour l'enseignement des sourds-muets, in Bulletin administratif de l'Instruction publique, 14, 166, p. 380-381.

Duruy, V. (1866) Circulaire à MM. Les Recteurs sur l'enseignement des sourds-muets admis dans les écoles primaires, in Bulletin administratif de l'Instruction publique, 5, 97, p. 288-291.

Encrevé, F. (2012) Les Sourds dans la société française au XIXe siècle. Idée de progrès et langue des signes, Ivry/Seine : Créaphis Éditions.

Grau, É. (2003) La pédagogie associée, Connaissances et surdités, 6, nov. 2003, Paris : ACFOS, p. 28-29.

Hamm, M. (2010) L'apprentissage de l'écriture et de la lecture chez les personnes sourdes et malentendantes, Thèse de doctorat en sciences de l'Éducation, Strasbourg: Presses de l'A.N.R.T.

Hamm, M. (2012) La lecture chez quelques sourds lettrés, Les Dossiers des Sciences de l'Éducation, 28, p. $105-127$.

Institut royal des sourds-muets de Paris $(1827,1829,1832,1836)$ Circulaires regroupées sous le titre Les quatre circulaires, à toutes les institutions de sourds-muets de l'Europe, de l'Amérique et que l'Asie, in Renard, M. \& M. Renard (2015) Bibliothèque sourde, Les Essarts-le-Roi: Éditions du Fox.

Karacostas, A. (ed) (1990) Le Pouvoir des Signes, Paris: INJS de Paris.

Karacostas, A. (ed) (2001) Retour sur le congrès de Milan, Surdités, 4, décembre 2001, Paris: G.E.S.T.E.S. 
Karacostas, A. (2010) La souffrance des sourds au travail [en ligne], Cité de la Santé-Cité des Sciences. Disponible sur : http://www.cite-sciences.fr/fileadmin/fileadmin_CSI/fichiers/au-programme/ lieux-ressources/cite-de-la-sante/_documents/

766_2F389_2FConf_C3_A9rence_La_souffrance_des_sourds_au_travail.pdf (consulté le 21 juillet 2017)

Lachance, N. (2007) Territoire, transmission et culture sourde. Perspectives historiques et réalités contemporaines, Laval: Les Presses de l'Université de Laval.

Lane, H. (1984) When the Mind Hears. A History of the Deaf, NY: Random House ; trad. française de J. Henry (1991) Quand l'esprit entend. Histoire des sourds-muets, Paris: Odile Jacob.

L'Épée, C.-M. de (1776) Institution des sourds-muets par la voie des signes méthodiques, première partie, réédité in Renard M. \& M. Renard, Bibliothèque sourde, Les Essarts-le-Roi: Éditions du Fox.

Menière, P. (1853) De la guérison de la surdi-mutité et de l'éducation des sourds-muets : exposé de la discussion qui a eu lieu à l'Académie impériale de Médecine, Paris: Germer Baillère.

Mottez, B. (2006) Les Sourds existent-ils ? Textes réunis et présentés par Benvenuto, A., Paris: L'Harmattan.

Moyse, D. (2008) Revendications identitaires et sélection prénatale, La Croix, 4 novembre 2008.

Padden, C. A. \& T. Humphries (1988) Deaf in America. Voices from a Culture, Cambridge Massachusetts: Harvard University Press.

Philippon-Pointereau, C. (2013) Comment penser l'accueil et la prise en charge des enfants Entendants de Parents Sourds en libéral ?, Mémoire pour l'obtention du diplôme de Capacité d'Orthophoniste, Nantes: Archives de la bibliothèque universitaire.

Quérel, C. (ed.) (2013) Surdité et Santé mentale. Communiquer au cœur du soin, Cahiers de SainteAnne, Paris: Lavoisier.

Rémon, M. \& M.-Fr. Sayde (2006) Ateliers d'écriture [en ligne], Connaissances Surdités, 16, juin 2006, Paris: ACFOS, p. 29-31. Disponible sur : http://acfos.org/wp-content/uploads/base_doc/ pedagogie/atelierecriture_revue16.pdf (consulté le 24 juillet 2017).

Renard, M. (2001) Surdités, accessibilité et illettrisme, Les Cahiers de l'Actif, mars-juin 2001, p. 69-80.

Renard, M. (2016) Un fils de Thot. Chroniques sourdes, Les Essarts-le-Roi: Éditions du Fox.

Timmermans, N. (2005) Le Statut des langues des signes en Europe, en coopération avec le Comité pour la réadaptation et l'intégration des personnes handicapées (CD-P-RR), Strasbourg: Éditions du Conseil de l'Europe.

Yau, S.-C. (1992) Création gestuelle et débuts du langage. Création de langues gestuelles chez des sourds isolés, Paris: Langages croisés.

\section{NOTES}

1. Issues de diverses communautés sourdes, les LS sont multiples. Dans un effort d'harmonisation, elles finissent par se distinguer au national en ASL (American Sign Language), LSB (Langue des Signes Belge), LSF (Langue des Signes Française), etc.

2. Cuxac (1983), Lane (1984, trad. française 1991), Karacostas, $(1989,2001)$ parmi les premiers... 3. Bernard (2014), Encrevé (2012), Buton (2009)... Yann Cantin, historien sourd, spécialiste des Sourds-Muets à la Belle Époque, alimente en particulier un site riche et ouvert qu'il faut suivre. 
4. De Haerne (1804-1890), 1865, p. 10. La note 2 à la même page précise que l'initiative vient d'Alexandre Rodenbach, aveugle belge, élève de Valentin Haüy à Paris, auteur de Coup d'œil d'un aveugle sur les sourds-muets en 1829 - sourds-muets qu'il fréquentait, allant jusqu'à Lille rencontrer Jean Massieu qui y dirigeait une école spécialisée après son renvoi de l'Institut de Paris. En France, le principe de scolarisation pour tous date de 1882, mais les modalités d'application concernant les sourds sont reportées (Encrevé, 2012 : 327).

5. Langue Parlée Complétée, code manuel d'aide à la lecture labiale par discrimination des sosies labiaux.

6. Ses descendants transformeront l'orthographe du nom familial en Ménière.

7. Voir la belle collecte d'Yves Bernard (2014), à partir de sa thèse.

8. Au moment de la rédaction, en 1989, on célébrait alors le Bicentenaire des droits de l'Homme et de la mort de l'abbé de l'Épée.

9. Celle des Sourds engagés dans la défense de la LS et d'une culture propre, d'où la majuscule pour les distinguer des autres sourds et malentendants (Cuxac : 1983). Michèle Riot-Sarcey, dans sa préface au livre de Florence Encrevé, souligne que le sentiment communautaire est loin d'être partagé par tous les sourds. Il ne s'exacerbe que dans le regroupement institutionnel et associatif. 10. Deux langues pour l'Éducation, association fondée en 1979 par des parents d'enfants sourds, des professionnels et des citoyens sourds.

11. Voir Domenjou. \& Laborit, Des psychiatres et des sourds... et la psychanalyse, in Bouillon (1990 : 71-81) ; l'intervention de Daniel Abbou à l'émission de La Marche du Siècle, « Le peuple des Sourds ", 1992 ; l'enquête nationale sur La Détresse psychologique des personnes sourdes etc., conduite par l'UNISDA, son colloque de restitution en 2011 et son rapport en 2012 ; Quérel, dir. (2013), en particulier p. 96-97.

12. Remerciant Pierre-Emmanuel Dauzat de m'avoir signalé cet ouvrage.

13. La sénatrice Dominique Gillot avait avancé ce pourcentage dans son rapport de 1998 et s'en explique lors d'une conférence à l'Institut National de Jeunes Sourds de Paris le 1er octobre 2015 ("Le droit des sourds»: où en est-on? Dix ans après la loi de 2005, Dominique Gillot revient sur son rapport, en ligne sur le site du même Institut - consulté le 8 août 2017) : C'était le recensement que nous avions des enfants soit non scolarisés, soit scolarisés dans des établissements où ils n'avaient pas la maîtrise de la langue. C'était un chiffre extrêmement fort qui m'a été apporté par l'administration et par les associations. Mais je pense qu'aujourd'hui, ce chiffre n'est plus le bon. Il faudrait vraiment qu'il soit réactualisé. Parce que, depuis, l'enseignement de la langue et de la lecture a fait des progrès, et il y a de plus en plus d'enfants sourds qui apprennent à lire. Jean-Louis Brugeille, professeur sourd devenu Inspecteur académique, lui répond: Il n'y a pas de réalité scientifique, de chiffres, de recherche, de statistiques, on reste donc dans le flou avec ce chiffre. L'estimation reste contestable dès la rédaction du rapport et ce commentaire postérieur pas évident.

14. Terme prêtant à confusion, ce ne sont pas des textes administratifs mais des appels à contributions, des statistiques et des comptes rendus, publiés à l'initiative du baron Joseph-Marie Degérando - ou de Gérando, administrateur influent à l'Institut, qui venait de publier son grand œuvre, et dont le rapporteur principal, d'après Yves Bernard (2014), est son neveu, Édouard Morel, alors professeur dans le même Institut.

15. Comme c'était déjà le cas de Jacob Péreire auprès de sa sœur vraisemblablement, comme ce sera le cas au $\mathrm{xx}^{\mathrm{e}}$ siècle de nombreuses mères devenant orthophonistes, codeuses etc. d'abord pour leur enfant, ou de parents cofondant des classes, voire des écoles, avec l'aide de professionnels.

16. Mimique est un terme ancien et usuel, sans connotation péjorative a priori, pour désigner les signes créés spontanément par les hommes et par les sourds en particulier. Expressifs, d'imitation et d'action, ils sont rapidement accessibles. La convention établie par un usage collectif les modifie en partie et les intègre dans une syntaxe différente des langues orales (différence nuancée, voir Yau : 1992), donnant naissance aux LS. 
17. L'abbé Charles-Michel de l'Épée (1776: 77) emploie le mot de perroquet, promis à un bel avenir pour désigner les sourds oralisés, en 1776 : on retient pendant douze ou quinze mois un homme doué d'une âme raisonnable dans la classe des perroquets, en ne lui apprenant que des mots et quelques phrases des plus familières. Celui de singe est utilisé le plus souvent sous forme verbale, singer signifiant imiter sans être toujours péjoratif: geste imitant une action et son objet, ou même parole imitant un son. Voir par exemple Degérando (1827 : 93-94, 96-97) et l'extrait qu'il donne d'une lettre de Saboureux de Fontenay (ibid. : 316) : Nous sommes naturellement disposés à imiter ce que nous voyons; nous nous piquons, avec raison, d'être les singes de la nature. La langue exprime sans peine les sons dont les oreilles ont été frappées. La fable Le Singe et le Perroquet est attribuée à Ésope (de Benserade, Les Fables d'Ésope mises en françois..., Rouen, 1789: 120) et connaît toutes sortes de variantes jusqu'à Alphonse Allais.

18. Comme le rappelle encore récemment Nathalie Lachance (2007: 238) mais en montrant qu'il est au moins double et divergent: Ainsi, l'histoire de la surdité ne devrait pas tellement être étudiée à travers la dualité constante entre les méthodes oralistes et les méthodes gestuelles et les qualités respectives de chacune pour l'instruction, mais à travers la finalité de ces méthodes qui prônent, d'une part, la normalisation et, d'autre part, le développement complet de la personne.

19. Voir par exemple, dans la Troisième Circulaire (1832: 112-114), l'option choisie par Sigismond Buergel, directeur de l'Institution des sourds-muets à Breslau, en Silésie : il fait observer que les enfants ordinaires apprennent à parler avant d'apprendre à lire et à écrire, et il pense que ce fait est une raison concluante pour suivre la même marche avec les sourds-muets. [...] Lorsque l'élève sait prononcer tous les sons, M. Buergel passe à la seconde partie de l'enseignement, qui consiste à faire connaître les caractères écrits et imprimés, et enfin la lecture. Ou encore, l'alphabet de prononciation farfelu proposé par Charles Barbier (ibid. : 200-206), etc.

20. Prosper Menière, à vif, en rend compte dès son introduction (1853, p. XXIII) : jugements dictés par la haine, libelles empoisonnés, lutte dans laquelle la passion ne négligeait rien pour l'emporter sur le bon sens, la raison, la vérité.

21. L'auteur rappelle qu'il existait à l'Institut de Paris un conseil de perfectionnement jusqu'en 1839, ces réunions favorisant des échanges d'expériences et d'interdisciplinarité avant l'heure. Depuis sa suppression, chaque professeur agit à sa guise.

22. La circulaire de l'Éducation nationale du 3 février 2017 portant sur la « Mise en œuvre du parcours de formation du jeune sourd », BO EN, $n^{\circ} 8$ du 23 février 2017, précise en troisième partie que pour les élèves signants, une scolarité individuelle, sans pair avec qui échanger en LSF, peut constituer un facteur d'isolement et d'appauvrissement de la langue. Le tout nouveau PEJS (Pôle d'enseignement pour les Jeunes Sourds) convient mieux aux bilingues (LSF+ français) assurément, mais aussi aux élèves sourds oralisants qui vivraient mal l'isolement en milieu ordinaire. Pour ces derniers, le mal-être n'est pas toujours bien ciblé, tant ils s'efforcent d'adopter le comportement de leurs camarades entendants et se sentent valorisés d'être mêlés à eux. Une jeune collégienne sourde profonde, remarquablement oralisée, a pu s'exclamer avec horreur, quand, face à ses difficultés de suivi scolaire, il était envisagé de l'orienter vers des classes adaptées: Mais si je viens ici, je serais avec des élèves qui sont comme moi? (Entretien personnel en 2007 ; la décision mûrie sera prise en 2008). On a vu des jeunes sourds, aptes à poursuivre des études générales, mais souffrant d'isolement, vouloir s'inscrire en filières professionnelles uniquement pour rejoindre des camarades sourds.

23. Florence Encrevé (2012 : 199, notamment) explore ce hiatus entre égalité des moyens et celle des fins (ou des chances), la première conduisant à définir des incapacités et le besoin de protection (voire de tutelle). Avec le républicanisme de 1870, explique-t-elle, le contrôle centralisateur pour l'application d'une norme majoritaire s'impose.

24. Et l'auteur d'ajouter : mais par la langue ordinaire, jusqu'à quel point?

25. Voir notamment Baynton (1996). Remerciant à nouveau Pierre-Emmanuel Dauzat de m'avoir signalé cet autre ouvrage.

TIPA. Travaux interdisciplinaires sur la parole et le langage, 34 | 2018 
26. Voir par exemple l'article de Monica Davey dans le New York Times, mis en ligne le 21 mars 2005, http://www.nytimes.com/2005/03/21/us/as-town-for-deaf-takes-shape-debate-onisolation-reemerges.html (consulté le 24 août 2017). Laurent Clerc, devenu sourd, a été instruit par l'abbé Sicard à Paris et il a ensuite accompagné le pasteur Thomas Hopkins Gallaudet aux États-Unis pour fonder un institut spécialisé.

27. Alors que j'enseignais au Cours Morvan, un de mes élèves, saturé des efforts à fournir en milieu entendant et jusque dans sa famille, demanda lui aussi dans les années 1990 : un petit département réservé aux sourds, serait-ce impossible?

28. http://www.france5.fr/emissions/l-oeil-et-la-main/diffusions/09-09-2013_122534

29. Maison départementale aux Personnes Handicapées, décisionnaire dans la reconnaissance du handicap et la prise en charge d'aides.

30. À l'occasion d'une conférence-débat sur la "Souffrance des sourds dans le travail» prononcée le 10 décembre 2010 à la Cité de la Santé-Cité des Sciences ; cf. http://www.citesciences.fr/fileadmin/fileadmin_CSI/fichiers/au-programme/lieux-ressources/cite-de-la-sante/ _documents/766_2F389_2FConf_C3_A9rence_La_souffrance_des_sourds_au_travail.pdf

31. Qui, en proposant le choix d'un projet linguistique au lieu d'un projet pédagogique, a eu pour effet de relancer un clivage français oral/LSF et leur exclusivité.

32. Jean-Pierre Bouillon (1990 : 33) est sceptique face à certains linguistes affirmant qu'il est facile de passer de la LS au français écrit. À mon avis, ils abusent.

33. Mélanie Hamm fait un point général sur l'accès à l'écrit de ceux qui sont atteints de surdité à divers degrés, précisant que la LS ne favorise l'apprentissage de l'écriture qu'à un certain niveau, étant elle-même une langue sans écriture (2010:271), ce qui recoupe les avis des premiers instructeurs, mais qu'en même temps, l'écriture est un medium fréquent, quelle qu'en soit leur maîtrise. Elle cite des travaux plus spécifiques à partir de la LS (ibid. : 267 et sq. en particulier). Sur la base de son enquête, elle vérifie que le niveau de lecture n'est pas tant lié au niveau de surdité et au mode de communication qu'au milieu socio-culturel (2012: 105-127), rejoignant les observations de Marc Renard (2001:69-80). Constat qui vaut pour tous : la stimulation des connaissances et les échanges informatifs facilitent l'entrée en lecture, quelle que soit la langue de départ.

34. Observations faites au Cours Morvan (Paris $9^{\mathrm{e}}$ ). Voir également Rémon \& Sayde (2006 : 29-31), concernant les ateliers d'écriture qu'elles ont animés.

35. Prosper Menière, citant à diverses reprises le Dr Jules Guérin, (1853 : 63 notamment).

36. Ibid. : 221, citant Jean-Jacques Valade-Gabel, alors inspecteur de l'enseignement des sourdsmuets dans les institutions départementales de France et directeur honoraire de l'Institution impériale de Bordeaux : selon lui, tous entretiennent une répugnance pour la société des parlants.

37. Les langues du Nord s'enseignent en effet plus facilement que les autres au moyen de la vue, parce qu'elles sont plus rudes, et qu'on les articule plus vivement et avec des aspirations plus marquées (Carton, $1845: 137)$.

38. De Haerne, 1865 : 157-158. Qui mieux qu'un Belge, partagé entre la France et l'Allemagne, sa patrie adoptant déjà la partition des langues voisines, expose au mitan du XIX siècle les situations bigarrées et mouvantes dans les choix de méthodes, avec des commentaires frappés au coin du bon sens? Si l'on met de côté quelques traits d'époque, son analyse pointe à peu près tous les éléments qui agitent notre actualité. Il présente un historique dont il cite les sources de premières et secondes mains avant d'aborder la controverse qui oppose les deux principales méthodes, française et allemande, espérant leur conciliation pour compenser les politiques en faveur de l'intégration en écoles ordinaires. L'une des voies pourrait être celle empruntée par l'Institution de Groningue fondée en 1790 aux Pays-Bas par Henri Daniel Guyot, formé par l'abbé de l'Épée, qu'il nomme méthode néerlandaise. Le tout est complété d'annexes statistiques et de coûts comparés, dans la mesure prudente des informations qu'il a pu récolter. 
39. Conclusion au congrès qui s'y est tenu en 1880, déséquilibré dans sa représentation et son déroulement, mais qui paracheva la victoire de l'oralisme et l'éviction des LS que concrétiseront une partie des pays participants. Voir Karacostas, dir. (2001), par exemple.

40. L'application de la méthode orale proscrivant la langue des signes en France n'est pas imputable au seul congrès de Milan. Elle est avant tout le résultat d'un processus déjà engagé (Encrevé, 2012 : 325).

41. Delaporte, 2016, p. 68 : au congrès des sourds-muets de 1898, par le directeur de Dijon, François Boyer; p. 247 : lors du discours du président de la mutualité en 1904 au Havre par Mr Fargeix, directeur d'école vouée à l'oralisme ; p. 273 : sœur Marie-Bernard traduit les sermons en signes jusqu'à sa mort (1948) à Angers...

42. Ce que nuance le Rapport Bouillon (1990: 270, par exemple) et d'autres en évoquant plutôt une crise, les échecs étant imputables à toutes les méthodes.

43. André Minguy lors de sa conférence à propos du Réveil sourd du 3 décembre 2014 mise en ligne sur http://etusourdes.hypotheses.org/100.

44. International Visual Theater, théâtre en LS fondé en 1976 par Jean Grémion et Alfredo Corrado, actuellement dirigé par Emmanuelle Laborit.

45. Une première à fortes répercussions en France et au-delà, dont naîtra deux ans plus tard à l'initiative du même concepteur, Jean-Marie Cavada, le premier hebdomadaire pour sourds et entendants L'œil et la Main, plus de dix ans après l'émission See Hear de la BBC ou Écoutez voir devenue Signes- en Suisse romande.

46. Dans l'enseignement, dans les années 1980-1990, on pouvait se servir de bases communicationnelles signées mais pas de lexique technique. En outre, les élèves eux-mêmes n'avaient pas du tout le même niveau de LS, ni de français. Jean-Louis Brugeille, alors enseignant sourd en collège, pointe le même problème en 2003.

47. Dominique Gillot, lors de son intervention à l'INJS en 2015 ("Le droit des sourds » : où en est-on? Dix ans après la loi de 2005), réaffirme cette primauté, mais sans l'oral : La langue d'enseignement, en France, c'est le français écrit. La vocation [...] de l'éducation, en France, c'est que tous les enfants maîtrisent le français écrit. Il y a eu un mouvement, ces dernières années, pour faire reconnaitre les langues régionales. Et je suis hostile à ça. Parce que je pense que l'unification de l'enseignement, en France, ça doit être le français.

[...] Il y a eu un mouvement aussi pour savoir si classer la LS comme langue minoritaire n'aurait pas été un moyen de favoriser son apprentissage dans les écoles. [...] Dans certains établissements, [...] il pourrait y avoir des cours en LS, pour tous les enfants de l'établissement, pas que pour les sourds.

[...] Pourquoi est-ce qu'on n'impose pas l'apprentissage de la LS ? On n'impose pas non plus l'apprentissage d'une langue régionale ou minoritaire. La langue d'enseignement, en France, c'est le français.

48. Cette modalité bilingue est clairement expliquée dans la brochure de l'établissement, accessible par son site internet ou celui d'École et surdité, de même que dans une vidéo de 2015 (https://youtube/kxQISAMN01A).

49. Voir notamment Grau (2003 : 28-29) ; l'auteur, orthophoniste au CEOP, centre dont le but est de tendre vers le bilinguisme - français oral/écrit-LSF - le meilleur possible, en fonction des possibilités de l'enfant, présente la pédagogie associée.

50. Voir en 2007 la riposte de certaines associations de sourds en Grande-Bretagne, réclamant le même droit à sélectionner les embryons porteurs de surdité dans l'article de Danielle Moyse, 2008. En remerciant Catherine Riou-Labrusse de me l'avoir signalé.

51. Illustré notamment par le mémoire d'Alexandre Graham Bell publié en 1884.

52. Tels Frankie Wilde ou David Guetta qui a déclaré sa surdité sur le mode d'un coming out... En miroir inversé, on a pu voir certains sourds des années 1990 faire leur autocritique devant leurs pairs pour avoir porté des appareils et suivi une rééducation à la parole, victimes de leurs parents ou des médecins... Une honte renvoie à l'autre, celle d'être sourd par rapport aux entendants ou celle d'être appareillé et de parler par rapport aux sourds signeurs. 
53. On relève déjà dans la seconde circulaire de Victor Duruy ce terrible point $n^{\circ} 3$ qui recommande de donner au jeune sourd des images, des mots et des phrases à mettre en correspondance : Grâce à ce moyen, il peut s'occuper seul pendant de longues heures.

54. Le succès dépend beaucoup plus de la pratique des équipes éducatives et des progrès de la technologie médicale que de la modification des textes, écrit Jean-Pierre Bouillon (1990:2). Ce sera vrai aussi pour le bilinguisme, la technologie informatique et multimédia en substitution...

55. Postface de Christian Cuxac au livre de Florence Encrevé (2012:360) : « Ne pas s'indigner, mais agir ".

56. Marie-Françoise Domenjou et Jacques Laborit écrivaient : La parole ne vaut qu'adressée à l'autre, ce n'est pas un produit pur, dans Des psychiatres et des sourds... et la psychanalyse, in Bouillon (1990: 72).

57. Voros, Pour un enfant sourd dans tous ses droits, in Bouillon (1990:230): Nous avons toujours refusé de lui enseigner une langue (quelle qu'elle soit); son langage s'est développé en situation de communication. Son langage à elle était un mélange de LSF, de français signé, d'oral et de mimique, toujours pour être le mieux comprise. Magali a très vite pris conscience de ses deux mondes.

58. Préface d'Alexis Karacostas, in Lachance (2007 : XVI).

59. Des orthophonistes ont obtenu des équivalences d'enseignement spécialisé pour le premier degré, mais au Cours Morvan, collège \& lycée d'enseignement général, privé sous contrat, certains orthophonistes ont cumulé certificat d'orthophonie et diplôme classique d'enseignement en lettres ou en philosophie. Ajoutons que l'orthophonie ne se borne pas au travail de l'oral et que l'on peut se servir de la LS.

60. Comme nous l'avons vu (supra p. 9, §26), les professeurs sourds sont progressivement puis définitivement remerciés en 1887 dans les instituts nationaux puisqu'il faut parler à tous les élèves sourds, les faire parler et les entendre. Depuis peu, ils peuvent postuler à la certification par la voie du BOE (Bénéficiaire de l'Obligation d'Emploi) au titre du handicap, en ayant les diplômes requis (master) ou une expérience, sans avoir à passer les écrits et les oraux du CAPES, mais en validant une année de stage qui comprend également la rédaction d'un dossier. Le CAPES de LSF a un statut particulier...

\section{RÉSUMÉS}

Les études sur l'histoire des langues des signes (LS) se concentrent sur le XIXe siècle, lorsque l'instruction s'est généralisée en Occident et que se développait celle des sourds. Les diverses institutions spécialisées, rassemblant les jeunes sourds, ont favorisé le développement des LS. La remise en cause de ces institutions, assimilées à des ghettos qui ne préparent pas l'intégration sociale et ne remplissent pas la mission d'instruction attendue, amplifie celle des LS, ciblées dès le début par les tenants de méthodes orales. À leur tour, ces dernières, s'imposant de la fin du XIXe siècle aux années 1980, sont accusées d'empêcher les sourds de s'approprier naturellement une langue, d'en faire des illettrés en difficulté d'insertion sociale et d'alimenter leur mal être.

L'écho récurrent, à plus de cent cinquante ans d'écart, d'un questionnement qui ne cesse pas entre LS ou oral, diversité des méthodes d'enseignement ou exclusivisme, instituts spécialisés ou intégration-inclusion, montre que le sujet est plus complexe que ne le pose en prémisse la focalisation sur l'un ou l'autre de ces paramètres. Une diversité et une souplesse revendiquées dès le XIXe siècle. 
Sign Languages (SL) asserted themselves and developed in some of the first specialized institutions for deaf people in Europe at the end of the $18^{\text {th }} \mathrm{C}$. and during the first half of the $19^{\text {th }}$ C. SL then began to lose ground with the growing demand for oral teaching and for the integration of deaf young people into regular schools. They also lost ground because the institutions for deaf people went through difficult periods (due to sharp criticism) and began to modify their educational project. In addition to that, towards the middle of the $20^{\text {th }} \mathrm{C}$., deaf pupils using SL started to be separated from those trying to speak. Oralism finally established, but experienced a crisis too in the late $20^{\text {th }} \mathrm{C}$. when SL came back on the scene and were practised anew.

It is difficult to study the deaf diaspora: censuses and evaluations of needs, as well as responses and outcomes, are not always reliable and neither were in the past. While the highlights and key players of this history are well-known, the transition periods are less well explored. These periods shed light on the complexity of the matter, and particularly on the training periods deaf people follow, whether the sign or speak. Even though the contexts, the moments, the people are different, the same issues can be raised from one century to the other. The tensions appearing as to the choice of an exclusive language for the deaf reflect the diversity of the goals to be achieved and also the diversity of the deaf people. In this paper, we will focus on the more balanced opinions-therefore "defeaned" by extremists of all sides-which give a warning against the danger of exclusivism. Indeed, since the 19th C., deaf people or hearing instructors as well as some physicians have been calling for the respect of diversity and warning against focalization on a single linguistic modality, method, purpose or anything that would reduce the scope of responses.

Abbot Carton (1845) and Canon Désiré de Haerne (1865), who practised in Belgium, reported that until the middle of the $19^{\text {th }} \mathrm{C}$., it was seen as a privilege to be admitted in an institution for deaf people: entries were limited, deafness taken into account and teaching adapted, whatever the method. Belgium represented at the time a sort of in-between France, where signs were prioritized, and Germany, where speech was prioritized, while mixed options (eclecticism) were adopted almost everywhere. Most deaf young people did not attend school at the time, especially as school became compulsory at different moments: 1836 in Belgium, 1882 in France...

In the second half of the $19^{\text {th }} \mathrm{C}$., and even more so from 1880 to the present day, the judgement was reversed. Separating deaf pupils who signed from deaf people who tried to speak resulted in assessing their intellectual capacities. The "best" pupils were placed in separate "articulatory" classrooms inside the institutes or even outside. At the same time "wild" integration in mainstream education persisted (it was later officialized with facilities for some pupils). Those having greater difficulty in speaking, reading, writing and understanding were declared unfit and kept in specialized institutions where care prevailed over education. Since the 1880s however, they have also been offered speech therapy courses.

Integration in mainstream schools slowly proceeded: by default first, because of the geographic dispersion of deaf children, their isolation and their limited enrolment in residential institutions. These conditions favored integration on a long-term basis, because most children had no other option, except preceptorship or no education at all. Secondly, integration was gradually valued by the prospect of a better education than in specialized institutions, and of socialization in the hearing world with a preparation for oral communication.

Specialized institutions still remained an alternative to the difficulties experienced in mainstream schools. But they began to transition away from sign education and definitively neglected it from the 1880s-even though deaf pupils as well as some teachers kept on using signs, despite the management prohibition. SL were thus openly practiced outside institutes in associations or meeting halls.

With the progress in speech therapy, hearing aids, Cued Speech (or LPC for Langue Parlée Complétée) and under the pressure of dissatisfied parents of deaf children who set up their own 
associations, the principle of integration in mainstream schools was promoted in the 1970s-1980s. At almost the same time, the Deaf Awakening emerged in France and Western Europe, following the Unites States of America, and swinging the pendulum back to SL, triggering the creation of bilingual classes (SL + national language). The advances of cochlear implants in the 1990s-2000s tipped the balance against in favor of oralism, but claims for bilingualism did not cease. LS were formally recognized, as well as the principle of bilingual education, although it was difficult to apply and took various forms.

Fears linger. Parents of deaf children are afraid of losing him/her due to a different mode of communication, a different identity and also fear the cruelty of social integration. Deaf people are frightened by technical advances promoting oralism. Hearing and deaf people share the same fear regarding early genetic detection, supposedly mutual pollution of SL and national languages, etc. It is important to reckon these fears so as to find appropriate responses to each. Sociologist Bernard Mottez wrote in 1977 a paper intitled persisiting against deficiencies amounts to increasing disability, in which he called to accept differences (or singularities) so as to preserve each individual's vital way of being. Oralized deaf, naturally or potentially bilingual, convincingly maintain that when diversity is respected, the bridges for reciprocal accessibility are easier to build. As for deaf signers, who now enjoy special facilities to validate qualifications and diplomas, they hold the key to biculturalism, which will be shared as long as communication spaces will be open.

Although some might regret its slowness, general accessibility to society has improved. Physician Prosper Menière, in 1853, had already understood the notion of inclusion, even before the word existed: "it is more difficult for deaf-mute people to make a step towards us than it is for us. We should move towards them". Inclusion, which lately replaced integration, implies society to adapt. This will only be possible if we get rid of the levelling equality which dissolves differences. The advocates of LS and deaf culture have been and still are accused of being closed off to progress because they are opposed to medical and paramedical repair which aims at conforming deaf people to the hearing-speaking majority. But it is this very medical tendency which generates a eugenic current that equates the deaf to deficient people or rejects them because of their disturbing difference. Deaf people nevertheless manage to take advantage of medical advances as well as technical progress in the field of communication (the Internet, visual communication systems and multimedia).

Finally, it is not the methods or the techniques that are questioned but the way they are applied. The adventure of LS and of deaf people in their diversity is above all a deeply human history, inscribed in situations of power and powerlessness, complex and moving on the surface of societal, scientific and technical streams at variable speed.

\section{INDEX}

Keywords : Sign Languages, Training Deaf, Deaf Education, Deaf History, Exclusivism, Eclecticism, Bilingualism, Oralism

Mots-clés : langues des signes, éducation des sourds, histoire des sourds, exclusivisme (en surdité), éclectisme (en surdité), bilinguisme, oralisme

\section{AUTEUR}

\section{AUDE DE SAINT LOUP}

Historienne et traductrice

aude.loup@gmail.com 